\title{
Prediction of Frost-Heaving Behavior of Saline Soil in Western Jilin Province, China, by Neural Network Methods
}

\author{
Xudong Zhang, ${ }^{1}$ Qing Wang, ${ }^{1}$ Zhensheng Huo, ${ }^{1}$ Tianwen Yu, ${ }^{1}$ Gang Wang, \\ Tianbao Liu, ${ }^{1}$ and Wenhua Wang ${ }^{2}$ \\ ${ }^{1}$ College of Construction Engineering, Jilin University, Changchun 130026, China \\ ${ }^{2}$ College of Civil Engineering of Changchun Institute of Technology, Changchun 130012, China \\ Correspondence should be addressed to Qing Wang; wangqing@jlu.edu.cn
}

Received 10 November 2016; Accepted 13 June 2017; Published 24 July 2017

Academic Editor: Francesco Pesavento

Copyright (C) 2017 Xudong Zhang et al. This is an open access article distributed under the Creative Commons Attribution License, which permits unrestricted use, distribution, and reproduction in any medium, provided the original work is properly cited.

In this study, backpropagation neural network (BPNN) and generalized regression neural network (GRNN) approaches are used to predict the frost-heaving ratio (FR) of the saline soil specimen collected from Nongan, Western Jilin, China. Four variables, namely, water content (WC), compactness, temperature, and content of soluble salts (CSS), are considered in predicting FR. A total of 360 pieces of data, collected from the experimental results, in which 30 pieces of data were selected randomly as the testing set data and the rest of the data were treated as the training set data, are applied to develop the prediction models. The predicted data from the models are compared with the experimental data. Then, the results of the two approaches are compared to obtain a relatively reliable model. Results indicate that the prediction model for the FR of saline soil in Nong'an can be successfully established using the GRNN method. Four new GRNN models are constructed for sensitivity analysis to assess the influence degree of the influencing factors, and the results indicate that water content is the most influential variable in the FR of the saline soil specimen, whereas content of soluble salts is the least influential variable.

\section{Introduction}

Seasonally frozen ground is widely distributed around the world. However, the seasonally frozen ground in Western Jilin is saline soil, which contains some soluble salts. Meanwhile, some of the soil in Western Jilin is dispersive soil [1], which is prone to many engineering problems, such as salinization, corrosion of the building foundation, frost heave, and thaw settlement. Western Jilin Province is a typical seasonal frost region, which is also one of the most severe salinization areas of China [2]. The ecosystem in this area is fragile, and the saline soil area increases year by year. Considerable research has been conducted to analyze the soil properties in Western Jilin. Zhang [1] collected soil specimens in Western Jilin and investigated its dispersing mechanism. Bao et al. [2] investigated the influencing factors of dispersive soil in Western Jilin using the gray correlation degree method. In previous research, the basic physicochemical properties [3], dispersion of soils [4], and hydraulic heat multiphases coupled geological environmental system of saline soil in
Western Jilin Province, China were investigated [5]. However, there is few literatures that studied the mechanism of salinization and frost heave; thus research on the engineering problems of saline soil in Western Jilin is insufficient. This study focused on studying the frost-heaving behavior of saline soil and the influence degree of the influencing factors. Soil samples were collected from Nong'an, Western Jilin, China, to assess the frost-heaving behavior of seasonally frozen ground.

In contrast to other kinds of seasonally frozen grounds, the soil collected from Nong'an, Western Jilin, is a typical saline soil. According to the rock soil engineering reconnaissance specification (GB50021-2001), the content of soluble salts (CSS) of saline soil is more than $0.3 \%$. In addition, according to previous research, the CSS of most soil samples was more than $0.3 \%$ in the study area, some of which were even approximately $1.5 \%$. Considering subgrade engineering and previous research [6], the frost-heaving ratio (FR) was measured and four parameters were selected for analysis, namely, compactness $(C)$, temperature $(T)$, water content (WC), and CSS. Modeling these factors using mathematical 
modeling and traditional process is complex. Thus, the neural network is assumed to be a feasible method to predict the FR from the design parameters.

The neural network method is inspired by the biological nervous system, which is widely used in engineering, image recognition, and voice recognition. The neural network was first applied in civil engineering in 1989 [7]. Then, the neural network method was used in many research subjects in engineering geology and civil engineering. The neural network has been applied in structural damage inspection [8], soft rocks strength prediction [9], ground vibration prediction [10], ground surface settlement [11, 12], engineering cost prediction $[13,14]$, concrete expansion prediction [15], soil swell potential prediction [16], triaxial compression behavior prediction [17], saturated hydraulic conductivity prediction [18], and other engineering subjects [19-21]. Only a fraction of the studies were about predicting the frostheaving behavior of saline soil in Western Jilin [22].

Many kinds of neural networks exist, such as backpropagation neural network (BPNN), discrete Hopfield neural network, radical basis function neural network (RBFNN), proportional-integral-derivative neural network, generalized regression neural networks (GRNN), and many other neural networks. Among these neural networks, BPNN is the most widely used and can be considered the most fundamental method [15]. Meanwhile, GRNN, which uses the probability density function, has robustness, a strong nonlinear mapping capability, and a high grade of error tolerance [23]. And previous researchers have been developed to compare the performance of these neural networks in application, especially for the BPNN and GRNN [23, 24]. Thus, these two methods (BPNN and GRNN) were used for predicting the frost-heaving behavior in this study.

The aim of this study is to present the methodologies for predicting the frost-heaving ratio of saline soil from Nongan, Western Jilin, and assess the influence degree of the four factors using sensitivity analysis. Computer programs were designed in MATLAB using BPNN and GRNN. The BPNN and GRNN models were developed and utilized to test the WC, $C, T$, and CSS using 360 available data. The BPNN model is a feedforward method, and the error algorithm in the BPNN network is backpropagation distribution. By contrast, the GRNN model is based on the RBFNN.

\section{Materials and Methods}

2.1. Experimental Materials. The saline soil collected from Western Jilin Province was used in this experiment. A sampling point was selected in the Nongan area in Western Jilin in November 2014 (Figure 1). A hole was dug vertically at the sampling point, and the soil was collected. Content of soluble salt of these soil samples is tested in the lab, and all the procedures abide by the specification for geotechnical test in China. The CSS of the soil at different depths, namely, 10,40 , and $70 \mathrm{~cm}$, were tested because salt migrates vertically with the variation of the seasons. The contents were $1.082 \%$, $1.392 \%$, and $1.224 \%$. The CSS of the $40 \mathrm{~cm}$ soil sample was the highest in the vertical profile of the superficial soil zone.

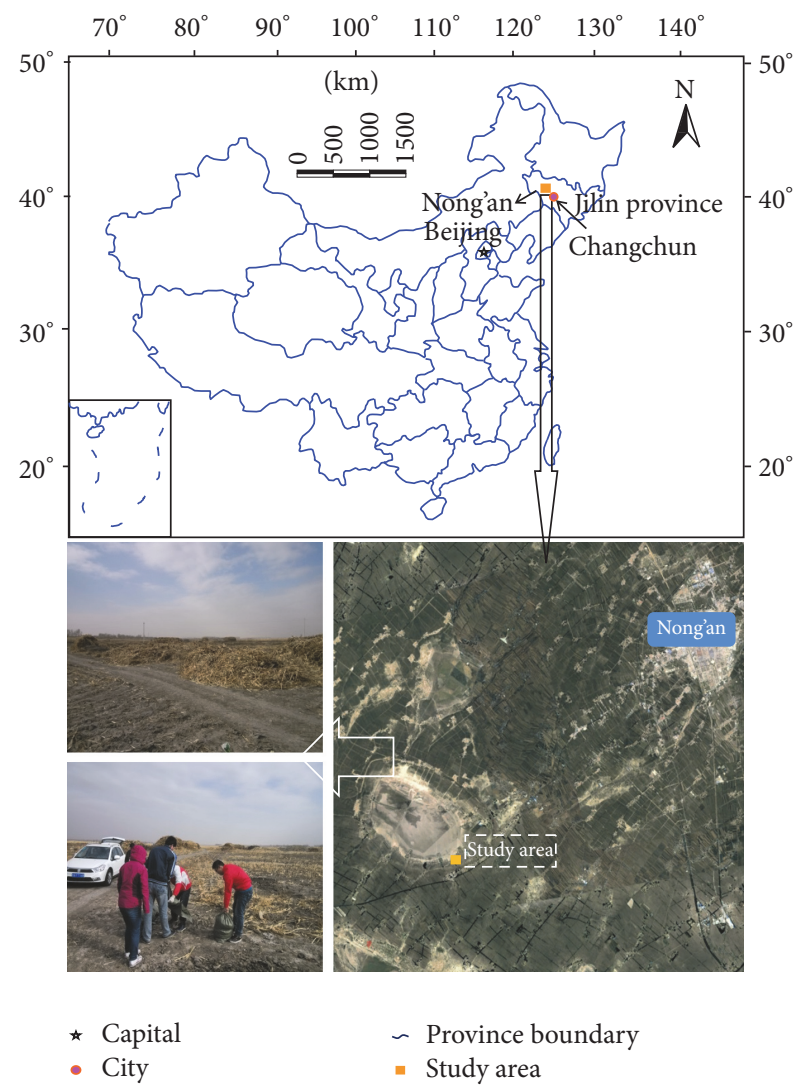

FIgURE 1: Geographical position of the study area, the position of sampling points.

Because the soil with salt may cause salt expansion, the soil of $40 \mathrm{~cm}$ depth may cause a relatively heavy frost heaving. In this study, the soil at the $40 \mathrm{~cm}$ depth was used for FR prediction. Soluble salts were added to some soil samples from the $40 \mathrm{~cm}$ depth after leaching by distilled water to control the variable CSS. According to the CSS in these three typical depths, the variable CSS was controlled. All the physical parameters and chemical composition of the soil at $40 \mathrm{~cm}$ depth and the designed CSS are shown in Table 1.

2.2. Specimen Design of Saline Soil. The large frost-heaving phenomenon of saline soil was reported in previous research $[6,22]$. Considering the frost-heaving effect, FR is defined as the ratio of the frost-heaving height and the original height of the specimen, which can be calculated by the following equation:

$$
\mathrm{FR}=\frac{\Delta h}{H_{o}},
$$

where $\Delta h$ is the frost-heaving height of the specimen and $H_{o}$ is the original height of the specimen.

The gray relational analysis method and rough set theory were used to identify the influencing factors of the FR [6]. The correlation degrees of cation exchange capacity, silt content, clay content, WC, specific surface area, CSS, $C$, and freezing $T$ were analyzed. Among these influencing factors, 
TABLE 1: Physical parameters and chemical composition.

\begin{tabular}{cccccccc}
\hline & $\begin{array}{c}\text { Granulometric } \\
\text { composition }\end{array}$ & Atterberg limits & Optimum & & & \multicolumn{2}{c}{$\begin{array}{c}\text { Chemical composition of } 40 \mathrm{~cm} \\
\text { soil samples and the designed } \\
\text { soluble salts samples (\%) }\end{array}$} \\
Silt (\%) & Clay (\%) & $\begin{array}{c}\text { Plastic limit } \\
(\%)\end{array}$ & $\begin{array}{c}\text { Liquid limit } \\
(\%)\end{array}$ & $\begin{array}{c}\text { Corresponding } \\
\text { maximum dry } \\
\text { density }\left(\mathrm{g} / \mathrm{cm}^{3}\right)\end{array}$ & $\begin{array}{c}\text { Content of } \\
\text { soluble } \\
\text { salts (\%) }\end{array}$ & $\begin{array}{c}\text { Designed } \\
\text { sample (1) }\end{array}$ & $\begin{array}{c}\text { Designed } \\
\text { sample (2) }\end{array}$ \\
\hline 81.37 & 18.25 & 17 & 51 & 21.26 & 1.63 & 1.40 & 1.08 \\
\hline
\end{tabular}

TABLE 2: Range of the variables.

\begin{tabular}{lcccc}
\hline variables & Variable properties & Maximum & Minimum & Range \\
\hline Compactness (\%) & Input & 95 & 85 & 10 \\
Water content (\%) & Input & 18 & 26 & 8 \\
Temperature $\left({ }^{\circ} \mathrm{C}\right)$ & Input & 5 & -25 & 30 \\
Content of soluble salts (\%) & Input & 1.40 & 1.08 & 0.32 \\
Frost-heaving ratio (\%) & Output & 2.297 & -1.750 & 4.047 \\
\hline
\end{tabular}

four strongly influenced the FR, namely, T, WC, C, and CSS. Meanwhile, the other four factors were eliminated in the evaluation and analysis of the influencing factors.

Parameters, such as WC, $C, T$, and CSS, should be designed to identify the influencing factors of the frostheaving effect and the degrees of each factor. In addition, almost all the aforementioned parameters can influence the frost-heaving behavior. Thus, specimens were designed according to the four variables to predict the FR of the saline soil specimen with mutually independent parameters.

FR changes with $T$. Moreover, these four parameters (WC, $C, T$, and CSS) were selected as the input variables to the BPNN and GRNN models. FR was selected as the output variable of the BPNN and GRNN models. The relationship between FR and its independent variables is characterized as follows:

$$
\mathrm{FR}=f(\mathrm{WC}, C, T, \mathrm{CSS}) .
$$

The optimum WC is $21.26 \%$. Five different WCs, namely, $18 \%, 20 \%, 22 \%, 24 \%$, and $26 \%$, were used to design the soil specimens. Three different grades of $C$, namely, $85 \%, 90 \%$, and $95 \%$, were used to design the specimens according to the maximum dry density. Eight different $T \mathrm{~s}$, namely, $5^{\circ} \mathrm{C}, 0^{\circ} \mathrm{C}$, $-2^{\circ} \mathrm{C},-4^{\circ} \mathrm{C},-8^{\circ} \mathrm{C},-12^{\circ} \mathrm{C},-20^{\circ} \mathrm{C}$, and $-25^{\circ} \mathrm{C}$, were used in the experiment. CSS varies with the depth of the soil samples. In this research, three typical depths of CSS were tested, selected, and applied in the experiment. The range of the variables is shown in Table 2.

2.3. Experimental Method. The specimens were $55 \mathrm{~mm}$ in diameter and $100 \mathrm{~mm}$ in height. Specimens in the experimental apparatus were placed in the temperature box immediately after preparation, as shown in Figure 2. $T$ gradually decreased from $5^{\circ} \mathrm{C}$ to $-25^{\circ} \mathrm{C}$ and was switched every $12 \mathrm{~h}$, which ensures that the FR can be stable under each $T$. The confined heaving ratio of every specimen was measured by the dial indicator each day until $T$ reached $-25^{\circ} \mathrm{C}$. Specimens with CSS ranging from $1.082 \%$ to $1.398 \%$ were fixed in the

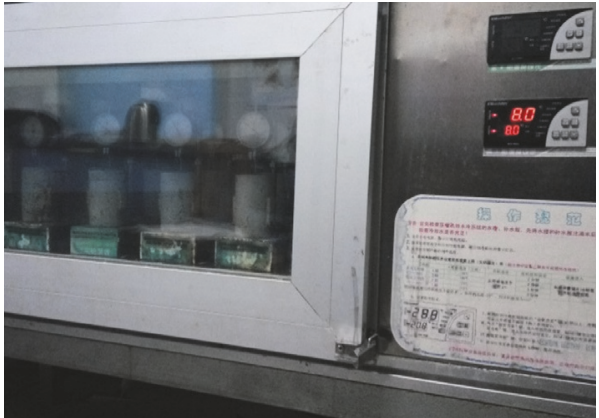

FIGURE 2: Experimental dies with specimen in the temperature box.

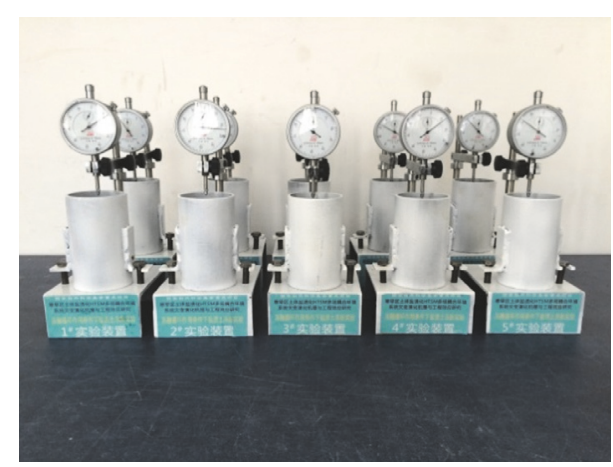

Figure 3: Experimental dies for the specimens.

experimental apparatus, as shown in Figure 3. The confined heaving ratio of all the specimens can be read from the dial indicator.

2.4. Development of the BPNN Model. The artificial neural network is a nonlinear adaptive mathematical system, which is based on the biological nervous system and composed of processing elements. Inspired by the biological nervous 
system, artificial neural networks have the capability of learning and remembering knowledge through the connection between neural elements [25].

Among the artificial neural networks, BPNN is used most widely and can be considered the most fundamental method. BPNN consists of three parts, namely, an input layer, several hidden layers, and an output layer. The input values of the design parameters are linked to the input neuron, and the values in the input neuron are linked to the hidden layer neuron by multiplying a connection weight [26]. Each value in the hidden layer is obtained by the summation $w_{i j} x_{i}$ and the deviation value $b$. All the hidden layer neurons weighted sums could be calculated using the aforementioned method, as follows:

$$
\text { (net) })_{j}=\sum_{i=1}^{n} w_{i j} x_{i}+b,
$$

where (net) ${ }_{j}$ is $j$ th hidden layer neurons weighted sum of the input layers, $x_{i}$ is the value of the $i$ th neuron of the input layer, $w_{i j}$ is the connection weight of the $i$ th neuron in the input layer and the $j$ th neuron in the hidden layer, and $b$ is the deviation value $[15,27]$.

Then, the value of the hidden layer output can be obtained using the following activation function:

$$
(\text { out })_{j}=f(\text { net })_{j}=\frac{1}{1+\exp \left(-\alpha(\text { net })_{j}\right)},
$$

where (out) ${ }_{j}$ is the $j$ th hidden layer output value, $f$ (net) ${ }_{j}$ is the activation function, and $\alpha$ is a constant term that refers to the slope of the function [28].

In this part, the BPNN model is constructed using the $\mathrm{BP}$ training algorithm to predict the confined FR of the specimens. Four input variables exist, namely, $C, \mathrm{WC}, T$, and CSS. All training and testing data were obtained from the previously described experiment. And the data are divided into two sets, in which 30 of the 360 pieces of data were selected randomly as the testing set data and the rest of data were treated as the training set data.

In addition, the experimental data in the models should be normalized using the following equation:

$$
X_{n}=\frac{\left(X-X_{\min }\right)}{\left(X_{\max }-X_{\min }\right)}
$$

where $X_{n}$ is the normalized data, $X$ is the data before normalization, $X_{\max }$ is the maximum data before normalization, and $X_{\text {min }}$ is the minimum data before normalization.

Only one hidden layer is set in this study, whereas the hidden neuron number could not be calculated in a reasonable method. However, the range of the hidden neuron number can be calculated in practice by the following function:

$$
n=\sqrt{n_{i}+n_{o}}+a
$$

where $n_{i}$ is the input neurons number, $n_{o}$ is the output neurons number, and $a$ is a modification value, which ranges from 0 to 10 . Thus, the hidden layer neuron number was determined to range from 3 to 13.

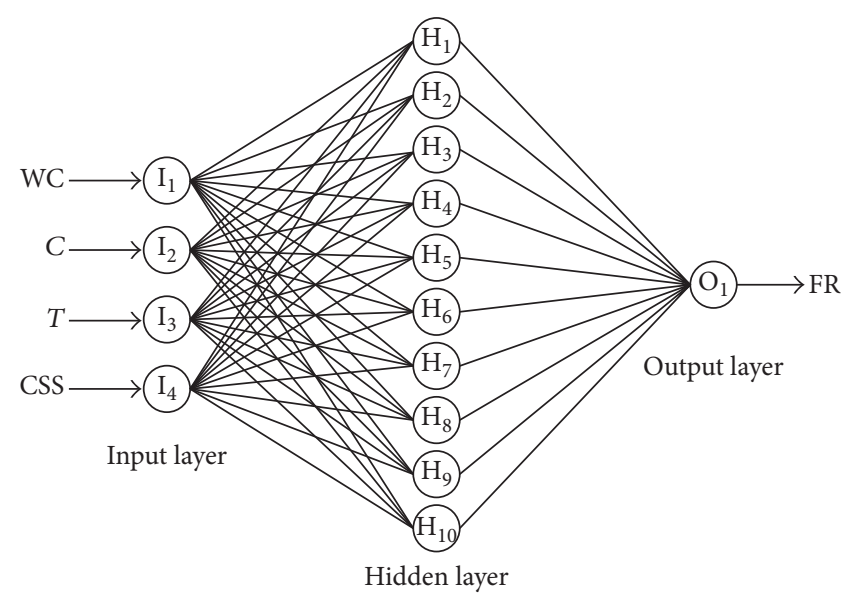

FIGURE 4: Structure of the BPNN model.

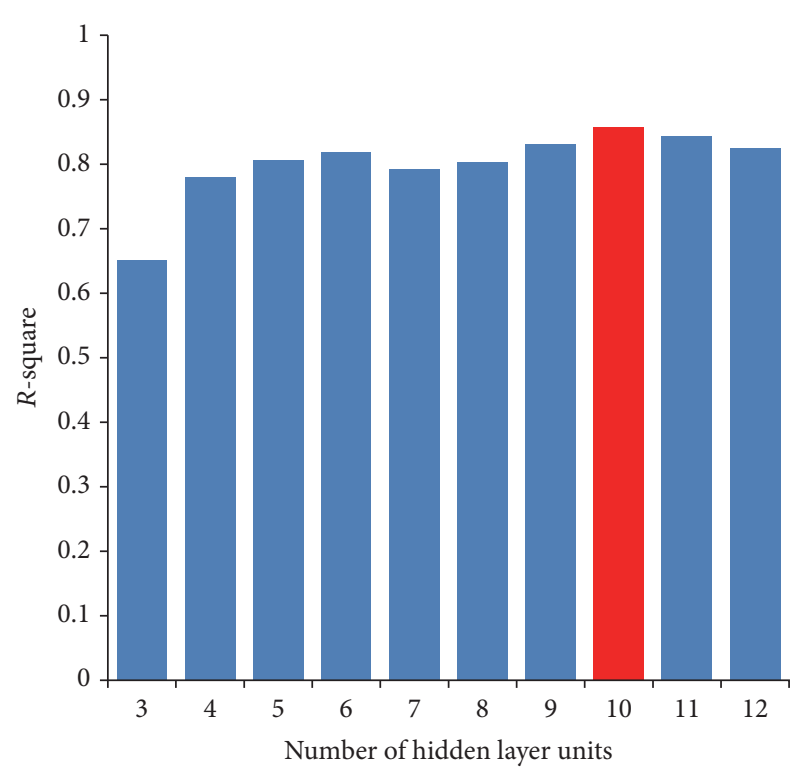

FIGURE 5: $R$-square value of the training data when the number of hidden layer units changes.

Three statistical parameters were applied to determine the proper neuron number, including $R$-square coefficient $\left(R^{2}\right)$, root mean square error (RMSE), and mean absolute percentage deviation (MAPD). A lower value is preferred for the RMSE and MAPD, whereas a higher value is preferred for $R^{2}$, which varies between 0 and 1 . The results of these three statistical parameters are shown in Figures 5, 6, and 7. The statistical parameters demonstrated that the best hidden neuron number is 10 . As a result, 10 neurons were selected in the model. The program is constructed using MATLAB to develop the BPNN model. Several parameters should be set. The number of input neurons is 4 and the number of hidden layer is 1 . The "tansig" function was applied in the hidden layer, whereas the "logsig" function was applied in the output layer. The BPNN model structure in this study is shown in Figure 4. 


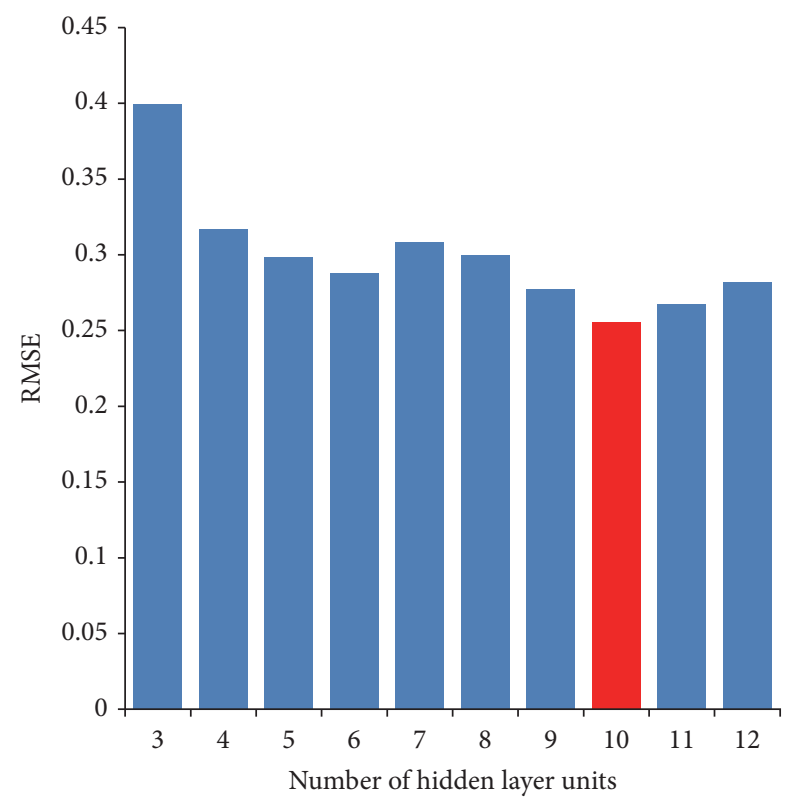

Figure 6: RMSE value of the training data when the number of hidden layer units changes.

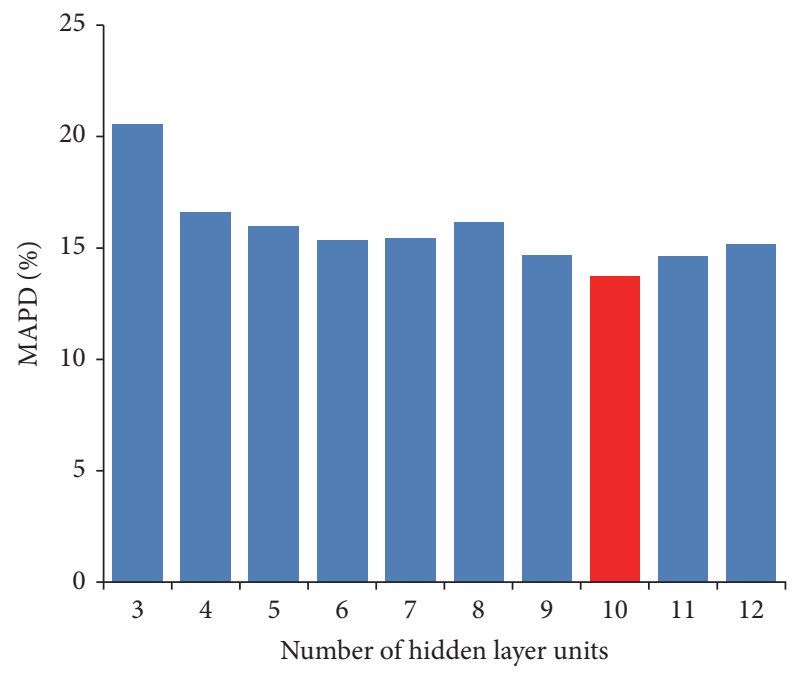

FIGURE 7: MAPD value of the training data when the number of hidden layer units changes.

2.5. Development of the GRNN Model. GRNN displays the difference of radial basis neural networks, which are developed from kernel regression networks [29-31]. In contrast to a backpropagation network, an iterative training procedure is unnecessary for GRNN. The vector of the input and output arbitrary functions can be remarkably close. The training data can help draw the function estimate directly [23]. Moreover, a substantiation analysis is conducted with many sampled data and training results, which indicate that the prediction has less error, with limited restrictions on the function [31]. The standard statistical technique applied in GRNN is called kernel regression. Unlike the RBF network, it is normalized with a hidden unit that consists of probability

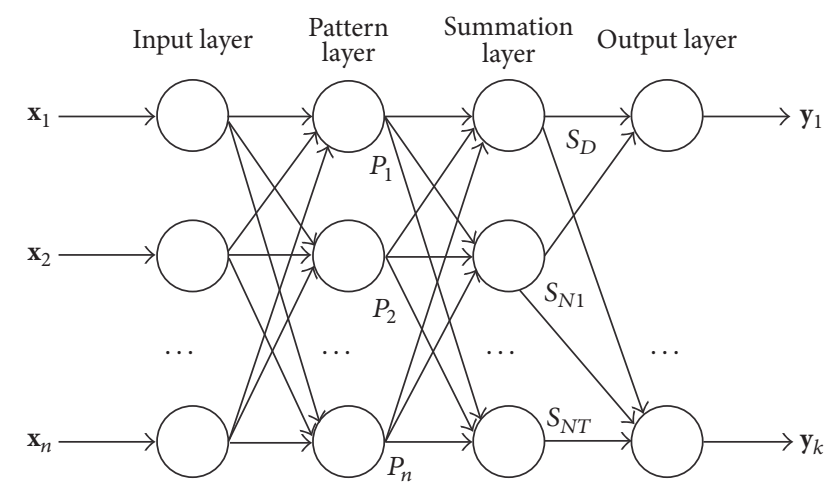

FIGURE 8: Structure of the GRNN model.

density functions at every training case. GRNN is a collection of four distinguishable layers, namely, input layer, pattern layer, summation layer, and output layer. The layers are shown in Figure 8. A training set can be given by estimating the joint probability density function between a dependent variable and an independent from GRNN method. This neural network theory is general, since the probability density function is obtained from the data without preconception of the form [24].

The number of observation parameters decides the unit number of the input layer. Every neuron connecting to the pattern layer shows a training pattern in the first layer. Two types of summations were presented in the summation layer, namely, summation and single division units [32]. The output set can be normalized by combining only the summation and output layers. The hidden and output layers will use the radial basis function and linear activation in the training process. $S$ and $D$ neurons in the summation layer have connections to each pattern layer unit. The weighted sum, which is related to the pattern layer, is computed by the $S$ summation neuron. Meanwhile, the pattern neurons unweighted outputs can be calculated by the $D$ summation.

Then, the output, which includes the results from the $S$ and $D$ summation neurons, can be divided by the output layer. Consequently, the predicted result $Y$ to the input vector $X$ is obtained as follows $[33,34]$ :

$$
Y=\widehat{f}(X)=\frac{\sum_{i=1}^{n} y_{i} \cdot \exp \left(-D_{i}^{2} / 2 \sigma^{2}\right)}{\sum_{i=1}^{n} \exp \left(-D_{i}^{2} / 2 \sigma^{2}\right)}
$$

where

$$
D_{i}^{2}=\left(X-X_{i}\right)^{T} \cdot\left(X-X_{i}\right)
$$

$n$ is the number of data samples, $y_{i}$ is the desired outputs, and $\sigma$ is the spread parameter. The larger the value of $\sigma$, the smoother the functional approximation. And when $\sigma$ is made small, some wild points would appear that may have a great effect on the estimate [35]. The optimal value of $\sigma$ must be determined experimentally depending on the application. 

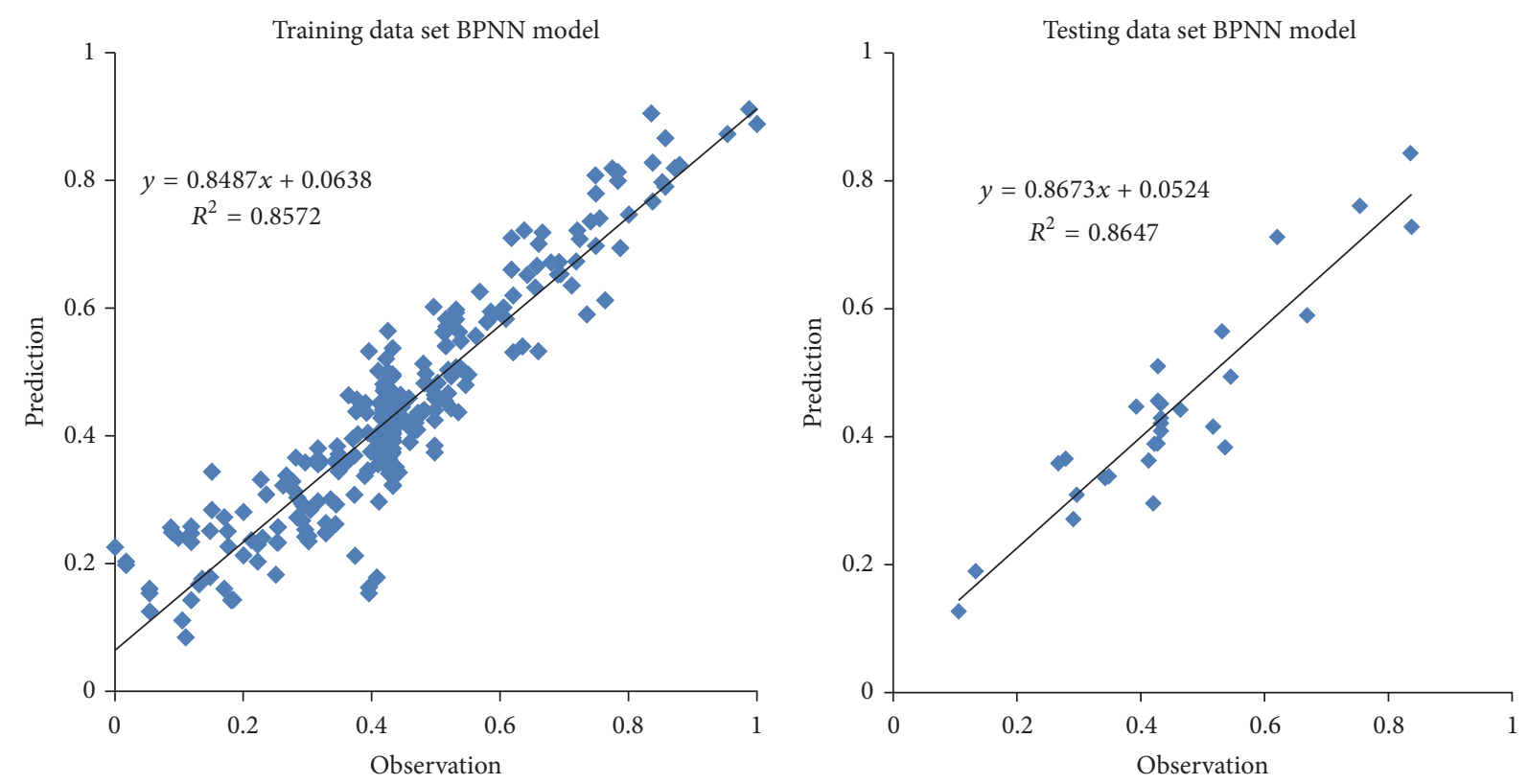

FIGURE 9: Relationship between observed and predicted FRs for the BPNN model.

\section{Results and Discussion}

Two models for predicting the FR of the saline soil specimens were developed. Three statistical indices were used to compare the models, as follows.

(1) $R$-Square Coefficient $\left(R^{2}\right) \cdot R^{2}$ indicates the fitting grade of the statistical models. $R^{2}$ varies between 0 and 1 , with 1 being the better value. Thus, the regression straight line fits the experimental data well if $R^{2}$ is close to 1 .

$$
R^{2}=1-\frac{\sum_{i=1}^{N}\left(\eta_{\mathrm{f}_{\text {_predict }}}-\eta_{\mathrm{f}_{\text {_experiment }}}\right)^{2}}{\sum_{i=1}^{N}\left(\eta_{\mathrm{f}_{\text {_predict }}}\right)^{2}},
$$

where $\eta_{f_{-} \text {predict }}$ is the predicted value of FR and $\eta_{f_{-} \text {experiment }}$ is the experimental data of FR.

(2) Root Mean Square Error (RMSE, [28]). RMSE can be computed to expose the error of the BPNN and GRNN models. RMSE is applied to evaluate the average differentials between the experimental value and the predicted results in the fitting line. A lower RMSE value is preferred. RMSE can be expressed using the following equation:

$$
\text { RMSE }=\sqrt{\frac{1}{N} \sum_{i=1}^{N}\left(\eta_{f_{\text {f_predict }}}-\eta_{f_{\text {f_experiment }}}\right)^{2}},
$$

where $\eta_{\mathrm{f}_{\text {_predict }}}$ is the predicted value of FR, $\eta_{\mathrm{f}_{\text {_experiment }}}$ is the experimental data of FR, and $N$ is the number of pieces of the data.

(3) Mean Absolute Percentage Deviation (MAPD). MAPD can be expressed using the following equation:

$$
\text { MAPD }=\frac{100 \%}{N} \sum_{i=1}^{N}\left|\frac{\eta_{\mathrm{f}_{\text {_predict }}}-\eta_{\mathrm{f}_{\text {_experiment }}}}{\eta_{\mathrm{f}_{\text {_predict }}}}\right| .
$$

Figure 9 shows the experimental data and the modeling results of BPNN, including the training and testing data. Figure 10 shows the experimental data and the modeling results of GRNN, including the training and testing data. In modeling the prediction, the values of the models are close to the experimental data shown in Table 3 and Figures 9 and 10. GRNN has better fitting result and lesser error than BPNN.

The three statistical indices of the regression models introduced previously were calculated in this study and are listed in Table 3. The training and testing $R^{2}$ values in the BPNN model were 0.86 and 0.86 , respectively. The training and testing RMSEs in the BPNN model were 0.26 and 0.25 , respectively. The training and testing MAPDs in the BPNN model were $13.71 \%$ and $12.28 \%$, respectively. Similarly, the training and testing $R^{2}$ values in the GRNN model were 0.96 and 0.87 , respectively. The training and testing RMSEs in the GRNN model were 0.14 and 0.25 , respectively. The training and testing MAPDs in the GRNN model were $4.27 \%$ and $12.44 \%$, respectively. The values of the three indices showed that the BPNN and GRNN methods have small deviations in the prediction of FR.

The $R^{2}$ value of the GRNN is closer to 1 in the training samples than that of the BPNN. In addition, the RMSE and MAPD values in GRNN are lower than those in the training samples. The statistical indices in GRNN are close to that in BPNN method in the testing data. Thus the results demonstrated that neural network methods are reliable in predicting the FR of saline soil in Nong'an, Jilin Province. And the GRNN method is superior to the BPNN method in this prediction. This is because the configurations between these two methods are different [23]. In simulations with BPNN integration, the algorithm is used to minimize output error with respect to the weights and thresholds and the optimum iterations are chosen with respect to the mean square error, while in the simulations with GRNN 

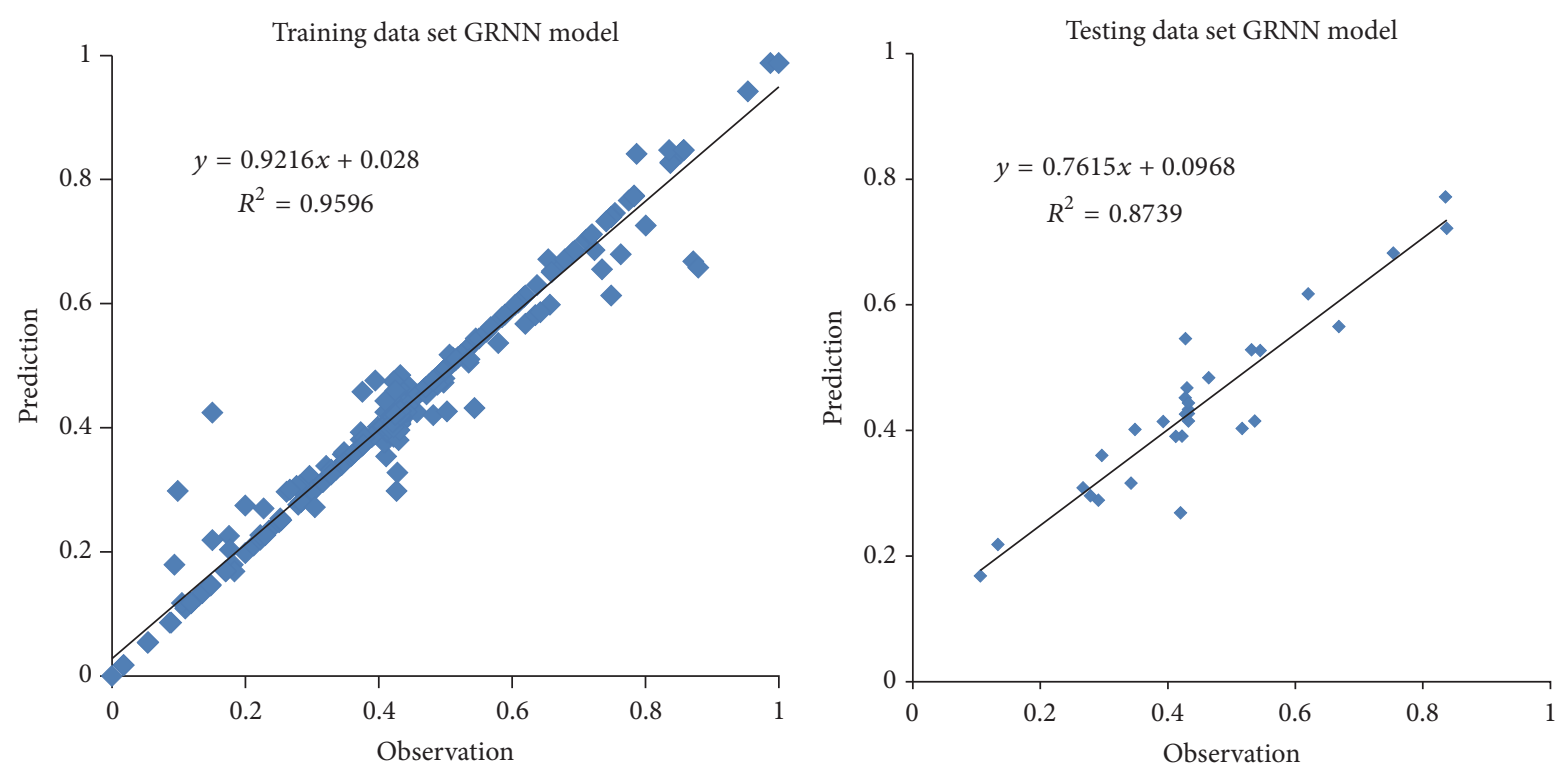

FIGURE 10: Relationship between observed and predicted FRs for the GRNN model.

TABLE 3: FR statistical value of proper BPNN and GRNN models.

\begin{tabular}{lcccc}
\hline Statistics parameters & BPNN & & & GRNN \\
& Training set & Testing set & Training set & 0.1404 \\
Testing set \\
\hline RMSE & 0.2552 & 0.2533 & 0.9596 & 0.2598 \\
$R^{2}$ & 0.8572 & 0.8647 & $4.27 \%$ & 0.8739 \\
MAPD & $13.71 \%$ & $12.28 \%$ & $12.44 \%$ \\
\hline
\end{tabular}

TABLE 4: Comparison of the performances of the GRNN models.

\begin{tabular}{|c|c|c|c|c|c|c|c|c|}
\hline \multirow{2}{*}{ Statistics parameters } & \multicolumn{2}{|c|}{ GRNN-I } & \multicolumn{2}{|c|}{ GRNN-II } & \multicolumn{2}{|c|}{ GRNN-III } & \multicolumn{2}{|c|}{ GRNN-IV } \\
\hline & Training set & Testing set & Training set & Testing set & Training set & Testing set & Training set & Testing set \\
\hline RMSE & 0.6285 & 0.6175 & 0.4181 & 0.2584 & 0.4646 & 0.4365 & 0.2880 & 0.3063 \\
\hline$R^{2}$ & 0.1494 & 0.3119 & 0.6392 & 0.8773 & 0.5437 & 0.6207 & 0.818 & 0.8028 \\
\hline MAPD & $23.86 \%$ & $25.19 \%$ & $19.98 \%$ & $11.85 \%$ & $21.40 \%$ & $20.75 \%$ & $12.53 \%$ & $12.13 \%$ \\
\hline
\end{tabular}

integration, the minimization of mean square error, the smoothing parameters, and the optimum iteration numbers are obtained according to the evaluated value. Several pieces of differences exist between these two methods: Firstly, in the prediction, the simulations in BPNN were required constantly until a satisfactory value of the performance criterion is obtained. Secondly, as is compared in previous studies, the processor time using the BPNN method is more than that using GRNN method in simulation [23,35]. Thirdly, in the learning process of BPNN method, the suffering local minima problem leads the model to a direction of inaccuracy.

3.1. Sensitivity Analysis. In this study, the influence degrees of WC, $C, T$, and CSS on FR are investigated. Four different types of models are constructed. The first model, called the GRNN-I model, includes $C, T$, and CSS as the input variables. The second model, called the GRNN-II model, includes WC, T, and CSS as the input variables. The third model, called the GRNN-III model, includes WC, C, and CSS as the input variables. The last model, called the GRNN-IV model, includes WC, $C$, and $T$ as the input variables. Then, the experimental data, which are divided into the training and testing sets, are trained and tested by these models. The statistical indices of these models are listed in Table 4.

Table 4 shows that the performance of the GRNN-IV model is better than that of the other models. In addition, the result of the GRNN-II model is better than that of the GRNN-III model and the CSS has the least influence on FR of the saline soil prediction model according to the indices shown in Table 4. Meanwhile, the influence of WC is the most significant because the prediction capability of the GRNN-I model, which does not include the variable $\mathrm{WC}$, is worse than that of the other models. Therefore, water content is the most influential variable on the FR prediction model. The influence degrees of the variables are as follows:

$$
\mathrm{WC}>\mathrm{T}>\mathrm{C}>\mathrm{CSS} \text {. }
$$

The comparison of these models is shown in Figure 11. 

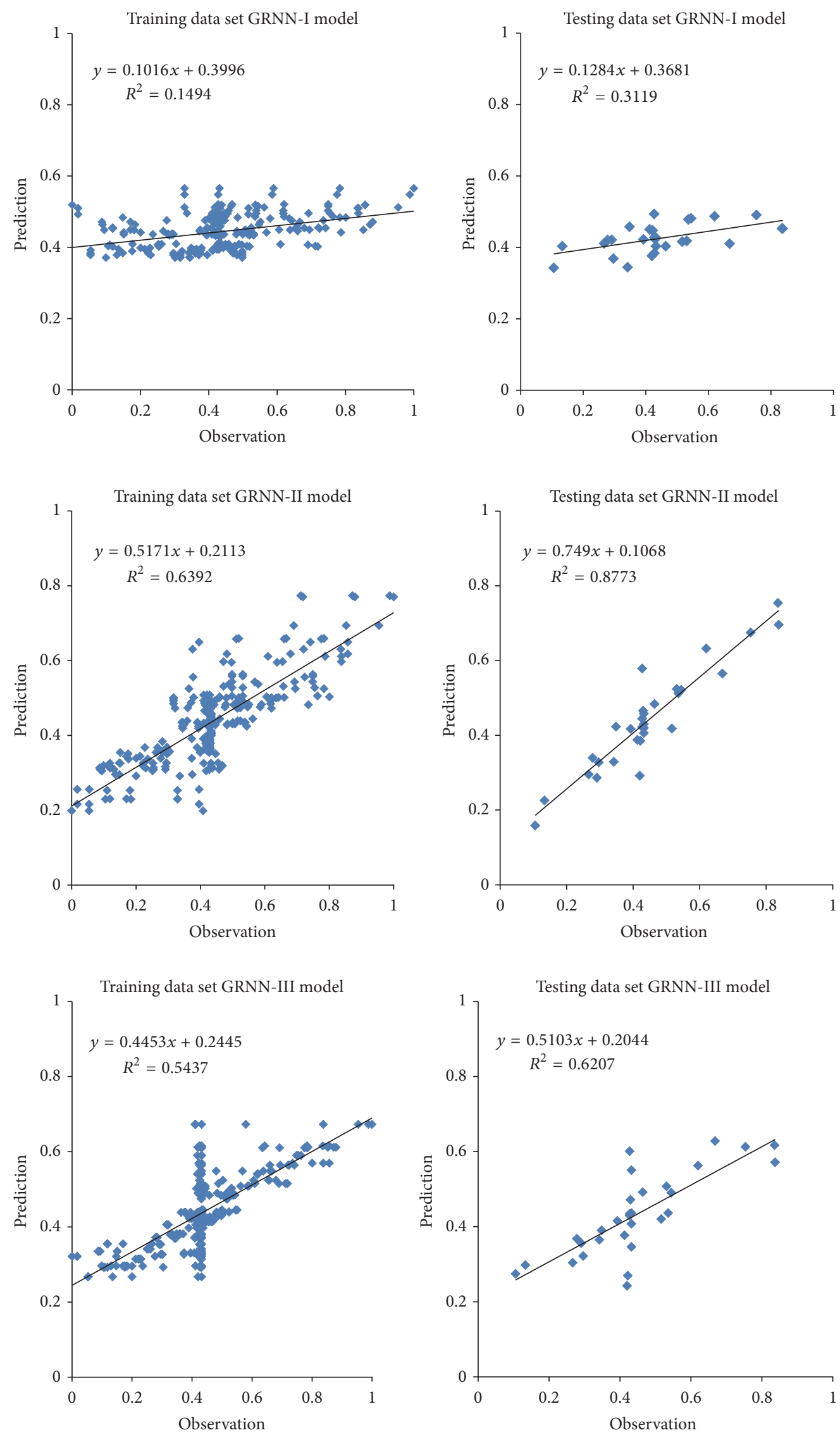

(a)

FIgure 11: Continued. 

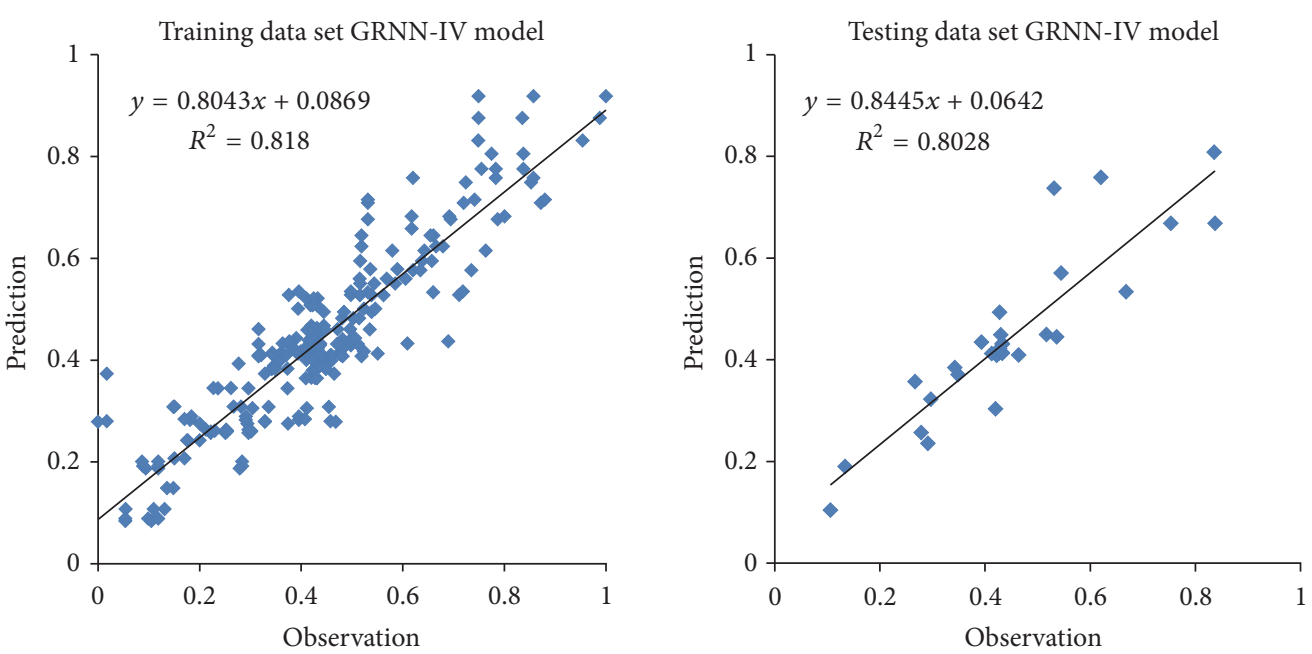

(b)

FIGURE 11: Relationship between observed and predicted FRs for the GRNN models.

In this research, the soil is carbonate-saline soil. The sensitivity analysis shows that soluble salts have the least effect on FR. The frost-heaving behavior of sulfate-saline soil and the saline soil with mixed salts will be investigated in future research to analyze the influences of different salts on frostheaving behavior and the mechanism of frost-heaving.

\section{Conclusions}

The FR of the saline soil specimen can be obtained using the BPNN and GRNN models, which means obtaining the proper frost-heaving ratio from water content, compactness, temperature, and content of soluble salt data. Therefore, BPNNs and GRNNs are suitable for predicting the FR of the saline soil specimen from Nong'an, Western Jilin.

In this research, the frost-heaving ratio of saline soil specimens with different water content, compactness, and content of soluble salt values were applied by the BPNN and GRNN models. The training and testing data samples in the models were the same. Meanwhile, most of the predicted values were close to the experimental result in the BPNN and GRNN models. The statistical indices, namely, $R^{2}$, RMSE, and MAPD, demonstrated that both methods are suitable for predicting the frost-heaving ratio of saline soil from Nong'an. Moreover, the GRNN model is more accurate than the BPNN model in this study. The predicted value and the experimental data indicated that GRNN is the appropriate method to adopt in establishing a reliable model for the prediction of FR.

Moreover, sensitivity analysis was conducted and four different types of models were constructed using the GRNN method based on the same data samples to determine the influence degree of the four input variables on the prediction model in this study. The result indicated that WC had the most effect on the FR, whereas CSS had the least. The sensitivity analysis shows that WC is the most influential variable on the FR of the saline soil specimens. The influence degrees of the variables are as follows: $\mathrm{WC}>T>C>$ CSS.

\section{Conflicts of Interest}

The authors declare that they have no conflicts of interest.

\section{Acknowledgments}

The authors are grateful for the financial support for the work reported in this paper from the State Key Program of the National Natural Science Foundation of China (no. 41430642) and the National Natural Science Foundation of China (no. 41372267 and no. 41302247). And the work is also supported by Graduate Innovation Fund of Jilin University (no. 2017067).

\section{References}

[1] J. Zhang, Research on the Dispersion Mechanism of the Dispersive Seasonal Frozen Soil in the Western of Jilin Province, Jilin University, Changchun, China, 2010.

[2] S. C. Bao, Q. Wang, and X. H. Bao, "Study on dispersive influencing factors of dispersive soil in western Jilin based on grey correlation degree method," Advance in Energy Science and Technology, vol. 291-294, pp. 1096-1100, 2013.

[3] T. W. Yu, Q. Wang, and X. D. Zhang, "Experimental study on grain size and soluble salt of saline soil in western Jilin Province, China," Sciences in Cold and Arid Regions, vol. 7, pp. 573-578, 2015.

[4] X. D. Zhang, Q. Wang, P. F. Li, and R. Y. Wang, "Research on soil dispersion of Qian'an soil forest," Journal of Northeastern University (Natural Science), vol. 36, no. 11, pp. 1643-1647, 2015.

[5] G. H. Yuan, The Mechanism and Numerical Simulation of Water Transfer in Seasonal Freezing Soil, Jilin University, Changchun, China, 2006.

[6] W. H. Wang, A Study on the Moisture Content Migration and Characteristics of Frost Heaving of Saline Soil in the Western of Jilin Province, Jilin University, Changchun, China, 2011.

[7] H. Adeli, "Neural networks in civil engineering: 1989-2000," Computer-Aided Civil and Infrastructure Engineering, vol. 16, no. 2, pp. 126-142, 2001. 
[8] X. Wu, J. J. Ghaboussi, and H. Garrett, "Use of neural networks in detection of structural damage," Computers \& Structures, vol. 42, no. 4, pp. 649-659, 1992.

[9] E. T. Mohamad, D. Jahed Armaghani, E. Momeni, and S. V. Alavi Nezhad Khalil Abad, "Prediction of the unconfined compressive strength of soft rocks: a PSO-based ANN approach," Bulletin of Engineering Geology and the Environment, vol. 74, no. 3, pp. 745-757, 2015.

[10] M. Hajihassani, D. Jahed Armaghani, A. Marto, and E. Tonnizam Mohamad, "Ground vibration prediction in quarry blasting through an artificial neural network optimized by imperialist competitive algorithm," Bulletin of Engineering Geology and the Environment, vol. 74, no. 3, pp. 873-886, 2015.

[11] C. Y. Kim, G. J. Bae, S. W. Hong, C. H. Park, H. K. Moon, and H. S. Shin, "Neural network based prediction of ground surface settlements due to tunnelling," Computers and Geotechnics, vol. 28, no. 6-7, pp. 517-547, 2001.

[12] S. Suwansawat and H. H. Einstein, "Artificial neural networks for predicting the maximum surface settlement caused by EPB shield tunneling," Tunnelling and Underground Space Technology, vol. 21, no. 2, pp. 133-150, 2006.

[13] C. M. Tam and C. F. Fang, "Comparative cost analysis of using high-performance concrete in tall building construction by artificial neural networks," ACI Structural Journal, vol. 96, no. 6, pp. 927-936, 1999.

[14] K. C. Sarma and H. Adeli, "Life-cycle cost optimization of steel structures," International Journal for Numerical Methods in Engineering, vol. 55, no. 12, pp. 1451-1462, 2002.

[15] B. Wang, T. Man, and H. Jin, "Prediction of expansion behavior of self-stressing concrete by artificial neural networks and fuzzy inference systems," Construction and Building Materials, vol. 84, pp. 184-191, 2015.

[16] I. Yilmaz and O. Kaynar, "Multiple regression, ANN (RBF, MLP) and ANFIS models for prediction of swell potential of clayey soils," Expert Systems with Applications, vol. 38, no. 5, pp. 5958-5966, 2011.

[17] D. Penumadu and R. Zhao, "Triaxial compression behavior of sand and gravel using artificial neural networks (ANN)," Computers and Geotechnics, vol. 24, no. 3, pp. 207-230, 1999.

[18] H. Merdun, Ö. Çinar, R. Meral, and M. Apan, "Comparison of artificial neural network and regression pedotransfer functions for prediction of soil water retention and saturated hydraulic conductivity," Soil and Tillage Research, vol. 90, no. 1-2, pp. 108116, 2006.

[19] M. Banimahd, S. S. Yasrobi, and P. K. Woodward, "Artificial neural network for stress-strain behavior of sandy soils: knowledge based verification," Computers and Geotechnics, vol. 32, no. 5, pp. 377-386, 2005.

[20] H. Bayat, E. Ebrahimi, and S. Ersahin, "Analyzing the effect of various soil properties on the estimation of soil specific surface area by different methods," Applied Clay Science, vol. 116-117, pp. 129-140, 2015.

[21] M. H. Baziar and Y. Jafarian, "Assessment of liquefaction triggering using strain energy concept and ANN model: Capacity Energy," Soil Dynamics and Earthquake Engineering, vol. 27, no. 12, pp. 1056-1072, 2007.

[22] S. C. Bao, Frost Heaving Characteristic and PFC-3D Numerical Analysis of Saline Soil in Western Jilin Province, Jilin University, Changchun, China, 2015.

[23] H. B. Celikoglu, "A dynamic network loading model for traffic dynamics modeling," IEEE Transactions on Intelligent Transportation Systems, vol. 8, no. 4, pp. 575-583, 2007.
[24] H. B. Celikoglu and M. A. Silgu, "Extension of traffic flow pattern dynamic classification by a macroscopic model using multivariate clustering," Transportation Science, vol. 50, no. 3, pp. 966-981, 2016.

[25] Z. Zhao and Y. M. Xu, Introduction to Fuzzy Theory and Neural Networks and Their Application, Tsinghua University Press, Beijing, China, 1996.

[26] F. Özcan, C. D. Atiş, O. Karahan, E. Uncuoǧlu, and H. Tanyildizi, "Comparison of artificial neural network and fuzzy logic models for prediction of long-term compressive strength of silica fume concrete," Advances in Engineering Software, vol. 40, no. 9, pp. 856-863, 2009.

[27] M. Saridemir, I. B. Topçu, F. Özcan, and M. H. Severcan, "Prediction of long-term effects of GGBFS on compressive strength of concrete by artificial neural networks and fuzzy logic," Construction and Building Materials, vol. 23, no. 3, pp. 1279-1286, 2009.

[28] M. Firat and M. Gungor, "Generalized regression neural networks and feed forward neural networks for prediction of scour depth around bridge piers," Advances in Engineering Software, vol. 40, no. 8, pp. 731-737, 2009.

[29] H. B. Celikoglu, "Application of radial basis function and generalized regression neural networks in non-linear utility function specification for travel mode choice modelling," Mathematical and Computer Modelling, vol. 44, no. 7-8, pp. 640-658, 2006.

[30] H. B. Celikoglu and H. K. Cigizoglu, "Public transportation trip flow modeling with generalized regression neural networks," Advances in Engineering Software, vol. 38, no. 2, pp. 71-79, 2007.

[31] H. K. Cigizoglu and M. Alp, "Generalized regression neural network in modelling river sediment yield," Advances in Engineering Software, vol. 37, no. 2, pp. 63-68, 2006.

[32] C. Suresh Kumar, V. Arumugam, R. Sengottuvelusamy, S. Srinivasan, and H. Dhakal, "Failure strength prediction of glass/epoxy composite laminates from acoustic emission parameters using artificial neural network," Applied Acoustics, vol. 115, pp. 32-41, 2017.

[33] B. Kim, D. W. Lee, K. Y. Park, S. R. Choi, and S. Choi, "Prediction of plasma etching using a randomized generalized regression neural network," Vacuum, vol. 76, no. 1, pp. 37-43, 2004.

[34] D. F. Specht, "A general regression neural network," IEEE Transactions on Neural Networks, vol. 2, no. 6, pp. 568-576, 1991.

[35] H. B. Celikoglu, "A dynamic network loading process with explicit delay modelling," Transportation Research Part C: Emerging Technologies, vol. 15, no. 5, pp. 279-299, 2007. 


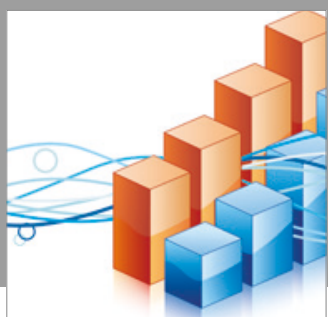

Advances in

Operations Research

vatersals

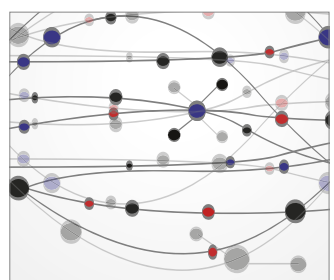

\section{The Scientific} World Journal
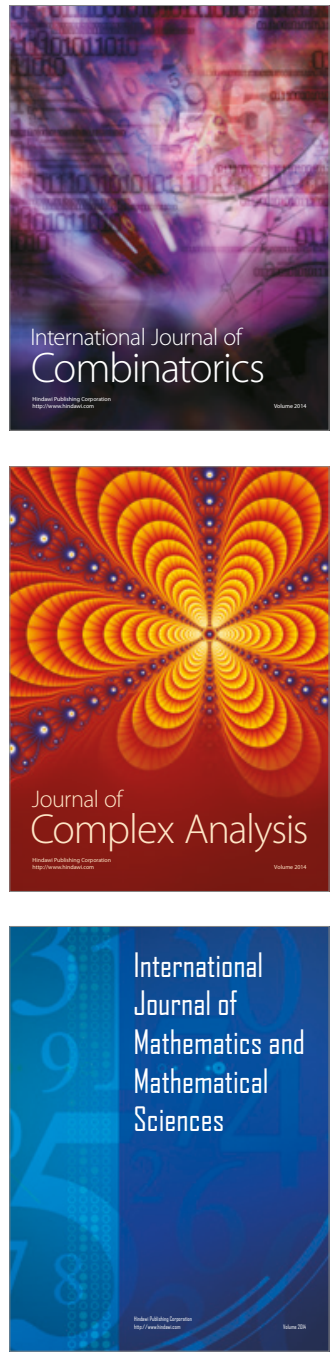
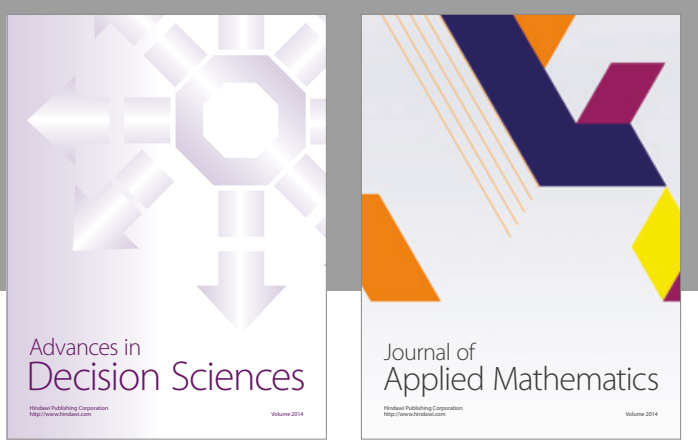

Algebra

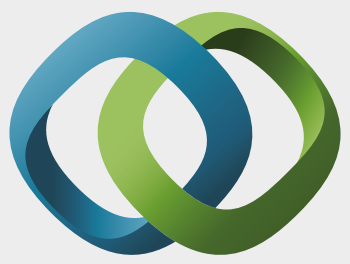

\section{Hindawi}

Submit your manuscripts at

https://www.hindawi.com
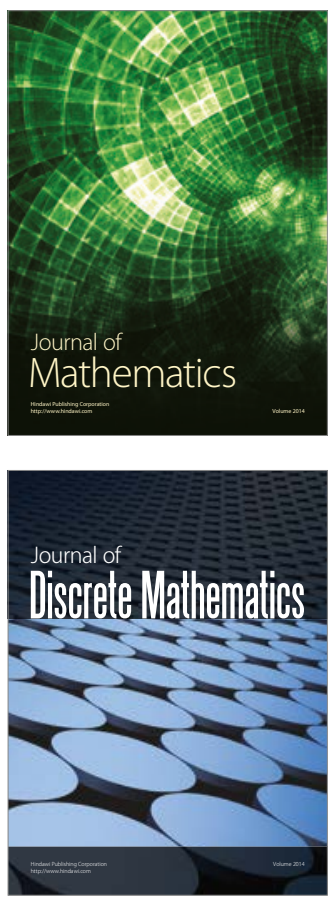

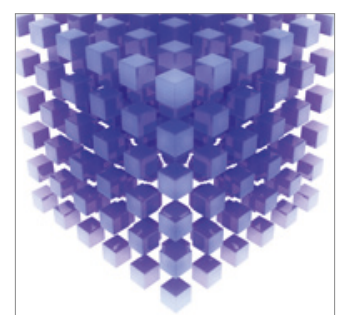

Mathematical Problems in Engineering
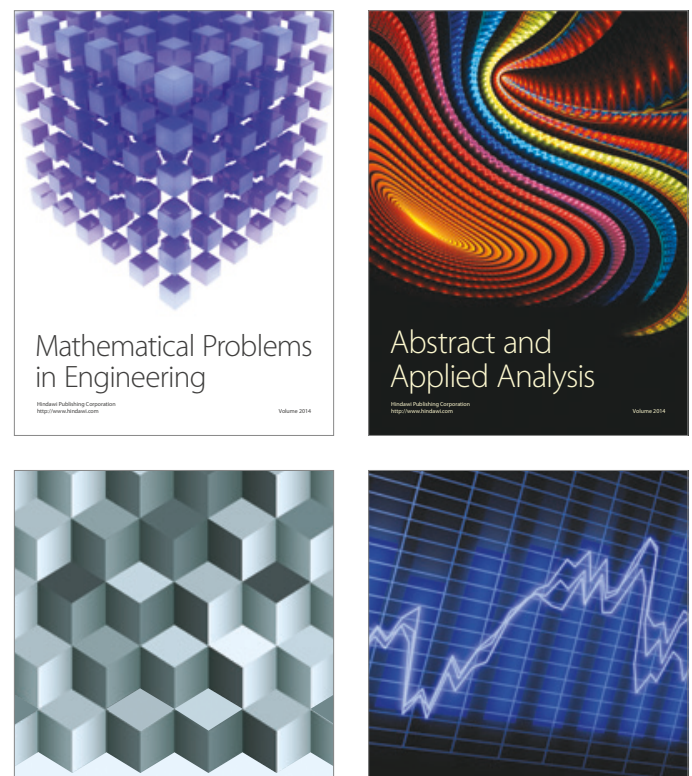

Journal of

Function Spaces

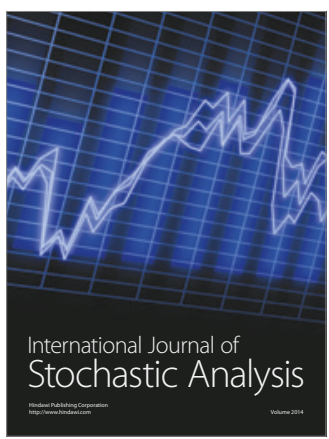

Probability and Statistics
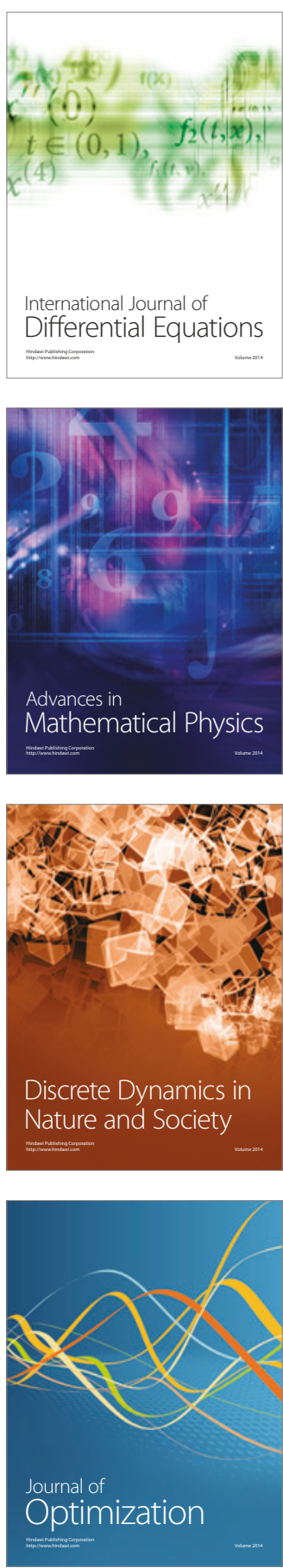